\section{Experiencias y percepciones de los graduados de Odontología sobre la culminación de la tesis en una universidad del Perú}

\section{Experiences and perceptions of dentistry graduates on the culmination of the thesis at a university in Peru}

\section{Artículo Original}

Yuri Castro-Rodríguez 1,a, Rosa Lara-Verástegui 2,b

${ }^{1}$ Pontificia Universidad Católica del Perú, Facultad de Educación, Lima, Perú.

${ }^{2}$ Universidad Nacional Mayor de San Marcos, Sociedad Científica de Estudiantes de Odontología, Lima, Perú.

a Magíster en Educación.

${ }^{\mathrm{b}}$ Bachiller en Odontología.

\section{Correspondencia:}

Rosa Lara Verástegui: rosa.lara1@unmsm.edu.pe Mz f Lte 52 Los Pinos, SJL. Lima. Perú ORCID: 0000-0003-4356-0297

\section{Coautor:}

Yuri Castro Rodríguez: yuricastro_16@hotmail.com ORCID: 0000-0002-9587-520X

\section{Editora:}

Angela Quispe-Salcedo

Universidad Científica del Sur, Perú.

Conflicto de intereses: los autores declaran no tener conflictos de interés.

\section{Resumen}

La tesis universitaria representa un trabajo académico donde el estudiante demuestra las competencias investigativas aprendidas; sin embargo, su culminación requiere habilidades específicas y sujetas a limitantes. Objetivo. Identificar las percepciones de un grupo de graduados de Odontología sobre sus experiencias al desarrollar su tesis de licenciatura. Métodos. Estudio fenomenológico que entrevistó a través de grupos focales a 32 estudiantes egresados de la Facultad de Odontología (Universidad Nacional Mayor de San Marcos, Lima, Perú) que culminaron satisfactoriamente su tesis de grado. Se exploraron las categorías de factores personales, relaciones e institucionales. El análisis se realizó de forma inductiva a través de las opiniones, búsqueda de similitudes y síntesis de hallazgos. Resultados. La responsabilidad laboral a futuro (factor personal) fue percibida como uno de los principales estimulantes para culminar la tesis; la motivación del asesor de tesis (factor relacional) fue percibida como una característica que influyó en la culminación mientras que el apoyo institucional y la consecución de datos (factores institucionales) fueron percibidas como condiciones negativas y difíciles de sobrellevar. Conclusiones. Diversos factores influyen en la culminación de la tesis universitaria como: la motivación del tema de tesis, el apoyo del asesor y contar con recursos para financiar la ejecución fueron características resaltadas como factores que promueven el desarrollo de la tesis universitaria. Estas experiencias se fundamentan en la obtención de un grado académico para insertarse en un sistema laboral.

Palabras clave: Investigación; Estudiantes; Percepción; Odontología; Educación (fuente: DeCS BIREME).

\begin{abstract}
The university thesis represents an academic work where the student demonstrates the research learned skills; however, its completion requires specific skills and subject to limitations. Objective. To identify the perceptions of a group of dental graduates about their experiences while the undergraduate students develop their thesis Methods. Phenomenological study that interviewed 32 students graduated from the Faculty of Dentistry (Universidad Nacional Mayor de San Marcos, Lima, Peru) through focus groups who successfully completed their degree thesis. The categories of personal, relationship and institutional factors were explored. The analysis was carried out inductively through
\end{abstract}

Fuente de financiamiento: no se contó con financiamiento para la realización del artículo original.

Recibido: $17 / 02 / 21$

Aceptado: $29 / 03 / 21$

Publicado: 01/07/21

( ) Los autores. Este artículo es publicado por la revista Odontología Sanmarquina de la Facultad de Odontología, Universidad Nacional Mayor de San Marcos. Este es un artículo de acceso abierto, distribuido bajo los términos de la licencia Creative Commons Atribución 4.0 Internacional (CC BY 4.0) [https://creativecommons.org/ licenses/by/4.0/deed.es] que permite el uso, distribución y reproducción en cualquier medio, siempre que la obra original sea debidamente citada de su fuente original. 
opinions, search for similarities and synthesis of findings. Results. Three categories of analysis were found: personal, relational and institutional factors. Future job responsibility (personal factor) was perceived as one of the main stimulants to complete the thesis, the motivation of the thesis advisor (relational factor) was perceived as a characteristic that influenced completion while institutional support and data acquisition (institutional factors) were perceived as negative conditions and difficult to cope with. Conclusions. Various factors influence the completion of the university thesis such as: The motivation of the thesis topic, the support of the advisor and having resources to finance the execution were characteristics highlighted as factors that promote the development of the university thesis. These experiences are based on obtaining an academic degree to enter a labor system.

Keywords: Research; Students; Perception; Dentistry; Education (source: MeSH NLM).

\section{Introducción}

En el contexto peruano, desde el 2014, la Ley Universitaria 30220 demanda que los estudiantes realicen una tesis universitaria para obtener grados académicos (sean estos bachillerato, licenciatura, maestría y doctorado) con el objetivo de fomentar la investigación en todos los niveles de la educación universitaria ${ }^{1}$. El desarrollo de este trabajo académico no es sencillo y se percibe como uno de los más difíciles de lograr. Las experiencias de los tesistas al culminar su trabajo de investigación han sido poco abordadas, principalmente en estudiantes de licenciatura. Los estudios realizados en doctorandos y maestrandos indican que culminar la tesis depende de factores personales, relacionales e institucionales ${ }^{2}$.

En la educación médica, las opiniones y experiencias de los graduados son importantes pues permiten identificar las falencias y condiciones favorables que viene desarrollando un programa para garantizar la calidad y seguir mejorando ${ }^{3}$. Monitorear la reacción de los estudiantes a sus experiencias de aprendizaje es una actividad que las instituciones de educación superior están emprendiendo cada vez más ${ }^{4}$. Este nivel de evaluación debe ser una característica inherente de todo programa porque ofrece formas en las que el programa se puede mejorar y desarrollar. La valoración y reacciones de los graduados de las ciencias de la salud sirven como un indicador de si el aprendizaje es posible.

Algunos estudios realizados en los programas de las ciencias de la salud indican que existen dificultades y percepciones negativas del proceso de elaboración de la tesis ${ }^{5,6}$. El estudio de los factores que influyen en estas experiencias permite otorgar información sobre cómo proporcionar apoyo y estructura a mejorar el proceso de la tesis. De esta forma, se sabe que los estudiantes más motivados con su tema de tesis culminarán en menos tiempo y sentirán que aprendieron más ${ }^{7}$, mientras que aquellos que perciban que existen problemas familiares, conflictos con la institución o interferencias laborales, demandarán más tiempo la culminación de la tesis ${ }^{8}$. Estos factores son complejos y varían de un estudiante a otro, por lo que la solución para la disminución de las barreras personales no es sencilla.

Poco se ha estudiado sobre estos factores en graduados del programa de Odontología, existiendo antecedentes en medicina humana ${ }^{9}$, higienistas dentales ${ }^{10}$ y estudios peruanos que se han centrado en la producción científica estudiantil y cómo se percibe la investigación ${ }^{11,12}$; de aquí que el presente estudio tuvo como objetivo describir las percepciones de un grupo de graduados de Odontología peruanos, sobre sus experiencias al desarrollar su tesis de licenciatura. El presente estudio se justifica en la necesidad de comprender mejor las limitantes de los tesistas y sus experiencias positivas que pueden ser replicadas en otras instituciones o con otros tesistas.

\section{Métodos}

Estudio cualitativo fenomenológico. La población fueron los estudiantes graduados de la facultad de Odontología de la Universidad Nacional Mayor de San Marcos (Lima, Perú) que culminaron su trabajo de fin de grado durante los años 2018-2020.

No se realizó cálculo muestral pues la intención fue obtener una comprensión profunda de un fenómeno que se conoce poco hasta conseguir una saturación teórica con las opiniones de los participantes ${ }^{13,14}$. Bajo este diseño se optó por incluir a 32 graduados que pudiesen participar de las entrevistas semiestructuradas. Se incluyó aquellos estudiantes que habían logrado sustentar su tesis universitaria de licenciatura y cuyos datos se encontrasen en el portal Cybertesis (https://cybertesis.unmsm.edu.pe/handle/20.500.12672/13). De este listado se envió correos electrónicos y mensajes a través de redes sociales para que participen en el estudio (82 invitaciones), no encontrándose respuesta en varios de ellos (50 graduados).

A los estudiantes se les invitó a participar del estudio a través de sus correos electrónicos y redes sociales. Se utilizó como técnica a la entrevista de grupos focales. El instrumento fue una guía de entrevista semiestructurada. Las categorías de las preguntas fueron: experiencia de la tesis, elementos facilitadores, motivaciones, tiempo de dedicación y recomendaciones para otros tesistas. Se realizó un piloto con cinco graduados para evaluar la claridad y el contenido de las preguntas; luego de esto, los graduados volvieron a participar de la ejecución junto con los demás participantes pues las preguntas fueron modificadas. 
La entrevista de los grupos focales contactados se realizó de manera virtual -debido a las restricciones sociales impuestas en el Perú por la pandemia de la COVID-19-, la investigación inició con la presentación del proyecto a los posibles participantes para obtener su consentimiento para la realización de la entrevista y agendar la fecha de la misma.

La guía de entrevista estuvo conformada por preguntas de datos personales (sexo, edad, trabajo actual y tiempo de duración de la tesis) y cuatro preguntas basadas en el estudio de Coruth et al. ${ }^{10}: 1$. ¿Cómo describiría su experiencia al completar la tesis? 2. ¿Cómo el proceso de la tesis le añadió valor profesional?, ¿qué cosas facilitaron el desarrollo de la tesis? 3. ¿Cómo describiría sus motivaciones para completar la tesis?, ¿qué barreras encontró al desarrollar su tesis? 4. ¿Cómo describiría el tiempo que tardó en completar la tesis? Basado en su experiencia, ¿qué recomendaría a los profesores y estudiantes para ayudar a completar sus tesis?

La entrevista se efectuó vía la plataforma Zoom. Al inicio de la reunión, se explicó la dinámica del estudio, el objetivo, las consideraciones éticas y se solicitó el permiso para grabar la sesión, indicando además que eran libres de abandonar la reunión en cualquier momento en caso de que ya no desearan participar del estudio. Los grupos focales fueron entrevistados por el mismo investigador para asegurar la coherencia en las preguntas formuladas a cada grupo, el cual realizó las preguntas a cada participante siguiendo el orden de las preguntas establecidas. Se mantuvo la privacidad y los participantes eligieron su nombre en la pantalla. Se realizaron cuatro grupos focales con ocho participantes cada uno, cada discusión duró entre una a dos horas. Al finalizar la entrevista se realizó las conclusiones y agradecimiento a cada participante.

El muestreo continuó hasta alcanzar la saturación de datos. El propósito de la saturación es asegurar que se recopilan suficientes datos de calidad para apoyar el estudio ${ }^{15}$. La saturación ocurre cuando los datos analizados llegan a un punto en el que no hay nueva información aparente. En el presente estudio la saturación se logró cuando se encontraron categorías en común y opiniones que se pudieron agrupar en dichas categorías. Cada grupo focal fue grabado en video para su posterior transcripción.

Para el análisis de la información, se transcribieron de forma literal colocando una nomenclatura a cada participante para su identificación ( $\mathrm{P}=$ participante). El investigador principal transcribió cada grabación dentro de las 24 horas posteriores a su registro; la transcripción se realizó en el programa Word a través de las opiniones de cada participante. Para asegurar la validez, la verificación de la información se llevó a cabo con una interpretación limitada de las opiniones de los estudiantes. Para la confiabilidad, otro investigador analizó de forma independiente las transcripciones y verificó la codificación. Una vez transcrita la información, se buscaron categorías y subcategorías temáticas y se compararon las respuestas intra e intergrupal. El análisis se realizó buscando factores personales, relacionales e institucionales según el enfoque inductivo de Liechty et al. ${ }^{2}$. Se identificaron los temas dominantes y se colocaron citas relevantes debajo de cada tema. La categorización de los datos crudos se realizó de manera manual. El presente estudio fue aprobado por el Comité de Ética de la Facultad de Educación de la Pontificia Universidad Católica del Perú como parte de la tesis doctoral del autor principal.

\section{Resultados}

Se enviaron cartas de invitación a 82 graduados de los cuales 32 aceptaron ser parte del estudio. El 62\% fueron mujeres, el promedio de edad fue de 28,5 $\pm 1,37$ años. A la fecha del estudio, el $55 \%$ de ellos se encontraba dedicado a la práctica clínica privada, y el promedio de tiempo que les demoró terminar la tesis fue de 1,7 $\pm 0,5$ años (Tabla).

Tabla. Características de los entrevistados

\begin{tabular}{ll}
\hline Características & $\mathbf{n}(\mathbf{\%})$ \\
\hline Sexo & \\
Femenino & $20(62)$ \\
Masculino & $12(38)$ \\
Total & $32(100)$ \\
Edad (años) & $28,5 \pm 1,37$ \\
Dedicación actual & \\
Práctica privada & $18(56,25)$ \\
Educación posgradual & $6(18,75)$ \\
SERUMS* & $4(12,5)$ \\
Otra & $4(12,5)$ \\
Duración de la tesis (años) & \\
Varones & $1,6 \pm 0,25$ \\
Mujeres & $1,8 \pm 0,75$ \\
Total & $1,7 \pm 0,5$ \\
\hline
\end{tabular}

*SERUMS= Servicio Rural Urbano Marginal en Salud

Las transcripciones de cada grupo focal permitieron identificar información clave para poder agruparlos en tres categorías: factores personales, relacionales e institucionales. Dentro de los factores personales se encontró expresiones y palabras relacionadas a: motivación, interés por el tema de tesis, apoyo familiar, dificultad del tema escogido, desconocimiento de procesos y tiempo de dedicación. Dentro de los factores relacionales se encontró expresiones y palabras relacionadas a: apoyo de amigos, gastos económicos, apoyo del asesor, críticas del jurado, clima de reuniones con el asesor y las competencias del asesor. Dentro de los factores institucionales se encontró expresiones y palabras relacionadas a: apoyo institucional, trámites administrativos, demora en los procesos, ambientes para la ejecución, acceso a fuentes de información, ambiente educativo, formación investigativa, y oportunidades de financiamiento.

Experiencia con la tesis y factores personales. Los graduados percibieron que su tesis fue motivante, el tema que escogieron les permitió profundizar en el estudio y que esto les motivó a culminarla. También indicaron que el hecho de obtener un título profesional es un factor motivante. "Culminar mi tesis y poder titularme para 
laborar de forma legal fue un elemento que me motivó" (P4). De manera general la experiencia que se tuvo de la tesis es que esta fue una buena experiencia de aprendizaje, que permitió a los estudiantes aprender temas relacionados a: búsqueda de la información, redacción académica, análisis de datos, pensamiento crítico, análisis de evidencia científica, elaboración de resultados y síntesis de la información. "Considero que con el desarrollo de la tesis aprendi elementos que no me quedaron claro en los cursos de metodología y tesis" (P12). Sin embargo, se tuvieron algunas barreras que limitaron el desarrollo de la tesis tales como: desmotivación en algunas etapas, desconocimiento de metodología y falta de tiempo por las actividades laborales. "No fue fácil conseguir la muestra en mi estudio, me demandó varios meses y a veces me quitaba motivación el tiempo que demoraba en la ejecución" (P21), "Desconocer de estadística dificultó el análisis de datos en mi tesis, tuve que contratar los servicios de un analista lo cual demandó más gasto" (P30), "Trabajar como asistente dental a tiempo completo dificultó el desarrollo de mi tesis pues hizo que no le dedique el tiempo suficiente ni la dedicación constante" (P14).

Para diversos graduados, el tema que escogieron fue lo más importante, pues la elección de un tema motivante facilita el desarrollo de la tesis. "Desde los cursos del pregrado que me gustaba la rehabilitación oral, el haber escogido un tema de tesis en este campo de estudio me motivó más" (P7). Otros graduados indicaron que sus temas no fueron motivantes y que los escogieron porque consideraron que iba a ser más "fácil". "Al inicio mi tema de tesis me gustaba, pero con el devenir de las lecturas me fui desmotivando, no lo comprendía muy bien... pero por la premura de querer obtener mi título profesional continué con ese tema" (P11). Otros participantes indicaron que escogieron temas que ellos consideraban "fáciles" al momento de ejecutar y que por ende culminarían más rápido la tesis; sin embargo, se dieron cuenta que al desarrollar y ejecutar el método no era tan fácil como ellos creían. Diversos graduados indicaron que la tesis debe entusiasmar al estudiante, que es una etapa donde el tesista debe disfrutar cada momento, cada lectura y cada discusión con el asesor/ jurado de tesis.

Experiencia con la tesis y factores relacionales. La experiencia de los graduados con los asesores de tesis no fue la más satisfactoria, pocos participantes indicaron que sus asesores apoyan el desarrollo de la tesis, otros indicaron que no ayudaron en nada, mientras que algunos indicaron que los asesores fueron un escollo a superar. "Recibi poca ayuda de mi asesor, al final casi todo lo tuve que hacer yo y las decisiones no las discutí con él" (P12), "Al inicio mi asesor si me ayudó otorgándome sus críticas e ideas, pero después ya casi ni le hablaba porque sabia que no me iba a responder" (P2). La ayuda y orientación del asesor no tuvo una experiencia favorable, aquellos que indicaron que sus asesores sí fueron muy rigurosos y críticos indicaron que se trató de docentes investigadores con amplia experiencia en sus líneas de investigación. "Mi asesor me guió en todo momento, él es investigador y sabia que cada ayuda que me daba era importante para mi formación" (P1). Este hallazgo indica una experiencia positiva del tesista.

Los gastos económicos que involucró la tesis fueron una limitante para algunos tesistas y un incentivo para otros (sobre todo para los que consiguieron financiamiento de sus tesis). "La ejecución de mi tesis fue muy costosa, requirió materiales e instrumentos que tuve que comprar pues no habia en la facultad, conseguir el dinero me demandó más tiempo" (P4), "Haber obtenido un financiamiento me permitió comprar materiales y costear el análisis estadístico, esto motivó mi avance" (P13). Los gastos fueron indicados principalmente al momento de ejecutar la tesis y durante el análisis de datos.

Para algunos tesistas fue importante el contar con el apoyo familiar ya sea para los gastos de la tesis, como agentes de escucha de las ideas del tesista, como consejeros y apoyando incluso más que el asesor. "El apoyo de mi familia a la tesis fue algo que lo hizo significativamente mejor, no tuve que preocuparme por buscar trabajo pues ellos comprendieron que primero tenía que culminar mi tesis" (P9). Un participante que tuvo una relación inadecuada con su asesor indicó: "Creo que mi asesor fue el menos útil en toda mi tesis. Casi nunca hablaba con él, más hablaba con mi familia sobre mis avances e ideas novedosas" (P18).

Percepción de la tesis y factores institucionales. Se encontró que el apoyo y orientación institucional sobre los procesos de la tesis fue deficiente y lento. Diversos graduados indicaron que su tesis pudo desarrollarse más rápido si la facultad hubiese tenido procesos más eficientes. "Los trámites de la tesis son muy lentos en la facultad, esto hacia que avance lentamente" (PG). También se indicó que la facultad adolece de espacios adecuados para desarrollar la tesis, principalmente en cuanto a laboratorios que cuenten con los instrumentos que permitan realizar mediciones. Se resaltó que muchas tesis se ejecutaron en los ambientes de la facultad y durante las actividades curriculares, para lo cual se contó con el apoyo de docentes y estudiantes quienes participaron en la ejecución. "Mi tesis requirió encuestar a los estudiantes, tuve que pedir permiso para entrar a los distintos semestres (promociones de estudio), afortunadamente los docentes y estudiantes me apoyaron" (P11).

Diversos graduados indicaron que existe una separación entre lo que se enseña en los cursos de metodología y tesis respecto a la tesis verdadera; es decir, percibieron que lo que se había enseñado no se ajustaba a la realidad. "En los cursos de metodología nos enseñaron algunos métodos y diseños, pero cuando uno realiza la tesis se encuentra con otros métodos que nunca te han enseñado" (P10). "Se me dificultó el tema de la validación del instrumento, pues en el curso de tesis nos indicaron que se realiza de una forma, pero mi asesor me indicó que debia hacerlo de otra forma" (P4). "Lo más complejo fue la redacción de la tesis, pues ni en los cursos de metodología o de tesis se nos enseña cómo redactar" (P5). 


\section{Discusión}

Completar una tesis requiere un compromiso de tiempo serio, implica lidiar con un jurado de tesis, aprender de un asesor y sobrellevar factores que pueden limitar su desarrollo (responsabilidades familiares, problemas de salud, actividades sociales, gastos, procesos administrativos, etc.) El presente estudio identificó las experiencias, valores percibidos, barreras, y motivaciones que tuvieron los graduados de Odontología cuando realizaron su tesis de licenciatura.

La tesis fue percibida como gratificante en el sentido que permitió aportar aprendizajes relacionados a la formación investigativa y profundizar un tema de estudio; sin embargo, se encontraron obstáculos y desafíos relacionados al tiempo de dedicación a la tesis, motivación del tema y saber cómo redactar los segmentos de la tesis. Esto ya había sido encontrado en el estudio de Ho et al. ${ }^{16}$ donde los tesistas indicaron que el desconocer cómo redactar limitada el avance de la tesis, de aquí que se recomiende que las facultades insistan en capacitar a docentes y tesistas en temas relacionados a redacción académica y científica, no solamente enfocarse en cursos de metodología o de seminario de tesis.

Nuestro estudio encontró que los graduados opinan que los procesos de la tesis no son claros y con la ejecución se aprende a sobrellevarlos, principalmente debido a una mala orientación ya sea por parte del asesor o de la institución; los hallazgos concuerdan con los estudios de Flynn et al. ${ }^{17}$ y Coruth et al. ${ }^{10}$ donde se encontró una limitada comprensión de los procesos por parte de higienistas dentales durante su posgrado. El análisis de las entrevistas de los grupos focales reveló un valor general del título profesional para continuar con los estudios de posgrado y obtener mejores oportunidades laborales. Los beneficios personales y laborales de los estudios de posgrado han sido valorados en diversos estudios ${ }^{18,19}$, los cuales no serían posibles si el tesista no culmina su tesis de licenciatura.

Los graduados percibieron que la tesis les ha permitido crecer profesionalmente y que un gran motivante fue el obtener un título profesional e insertarse al campo laboral. También se indicó que la elección de un tema de tesis motivante influye mucho en el desarrollo del estudio. Al respecto, Lundgren y Halvarsson ${ }^{20}$ encontraron que los estudiantes que estaban entusiasmados con su tema y sintieron que agregaron valor para su profesión evidenciaron una mejor experiencia al escribir una tesis que aquellos no presentaron estas características sin esos factores. Este hallazgo sugiere que los estudiantes son conscientes de los posibles beneficios profesionales el obtener un título y continuar con los estudios de posgrado.

El apoyo del asesor fue una característica considerada como limitante; diversos graduados percibieron que este apoyo fue poco crítico y en algunos casos fue nulo. Muchos expresaron tener un mejor aprendizaje con asesores que fueron motivadores, se mantuvieron positivos y mantuvieron al estudiante a tiempo para terminar la tesis a través de su liderazgo, orientación y entendimiento. Sin embargo, hubo participantes que experimentaron lo contrario. Barnes et al. ${ }^{21}$ acota que el asesor debe mostrarse accesible, ser amable, evidenciar empatía y cariño con su tesista para lograr que este disfrute el desarrollo de su estudio. Kumar et al. ${ }^{22}$ añade que esta relación asesor/estudiante es fundamental para lograr el avance de la tesis. Los estudiantes mencionaron que el recibir retroalimentaciones eficaces implica identificar aspectos positivos y negativos de su trabajo, esto se logra cuando el asesor y los jurados se vinculan con la tesis y la formación del tesista.

El apoyo decisivo del director de tesis (asesor/supervisor) es necesario en la construcción del estado de la cuestión y el marco teórico, ya que es en estas secciones donde el tesista encuentra más problemas. Lo mismo se puede decir sobre la revisión. Si tanto los directores como los estudiantes planean juntos las diferentes secciones de una tesis y si dedican algunas sesiones a conjuntar revisión, algunos errores ciertamente pueden evitarse.

Respecto a los factores institucionales se encontró que el apoyo de la facultad fue limitado y los procesos administrativos hicieron que la tesis demorase más. Aquí es recomendable que exista una orientación previa a la tesis y proporcionar detalles sobre el proceso como una forma de ayudar a los estudiantes a terminarla de una de manera oportuna. La falta de sinergia entre la parte académica y la gestión institucional puede limitar el avance de la tesis ${ }^{23}$, esto puede mejorarse con actividades de fortalecimiento, como la incorporación de un mayor número de docentes investigadores calificados acorde con las prioridades y líneas de investigación que la universidad promueve, y una mejor motivación en los estudiantes para potenciar su capacidad de investigación con la finalidad de generar conocimiento que aporte soluciones a los problemas de salud ${ }^{24}$.

El presente estudio no está exento de limitaciones y se puede indicar que se incluyó graduados que completaron satisfactoriamente su tesis, pudiendo existir un sesgo de autoselección por lo que se recomienda comparar los hallazgos con tesistas que aún no culminan su tesis o decidieron no ejecutarla. Los participantes fueron reclutados mediante muestreo no aleatorio y el tamaño de la muestra final fue relativamente pequeño. A pesar de que la tasa de participación fue moderada, no pudimos reclutar participantes de todos los años de egreso. Por lo tanto, los presentes resultados no pueden generalizarse a la población completa de graduados y pueden reflejar más que los resultados a largo plazo del programa.

Se concluyó que los graduados de Odontología percibieron como satisfactoria la culminación de la tesis universitaria; esta les permitió formar nuevas competencias investigativas. Este desarrollo no está libre de factores que promueven o limitan su culminación; se incluyen factores personales, relacionales e institucionales. La motivación del tema de tesis, el apoyo del asesor y contar con recursos para financiar la ejecución fueron características resaltadas como factores que promueven el desarrollo de la tesis universitaria. 


\section{Referencias bibliográficas}

1. Perú, Congreso de la República. Ley 30220: Ley Universitaria. Lima: Congreso de la República; 2014. Disponible en: http://www.minedu.gob.pe/reforma-universitaria/pdf/ley_universitaria.pdf

2. Liechty JM, Liao M, Schull CP. Facilitating dissertation completion and success among doctoral students in social work. J Soc Work Educ [Internet]. 2009 [acceso: 12/01/2021]; 45(3):481-95. Disponible en: https://www.tandfonline.com/doi/abs/10.5175/ JSWE.2009.200800091

3. Ochoa LS, Moreno EM. Some Perceptions of Master's Degree Students on the Comments (remarks) of their Thesis Directors. Signo y Pensamiento [Internet]. 2017 [acceso: 14/01/2021]; 36(71):188-206. Disponible en: http://www.scielo.org.co/scielo.php?script=sci_arttext\&pid=S0120-48232017000200188

4. Kahlon J, Delgado-Angulo EK, Bernabé E. Graduates' satisfaction with and attitudes towards a master programme in dental public health. BMC Med Educ. 2016 [acceso: 14/01/2021]; 15:61. Disponible en: https://doi. org/10.1186/s12909-015-0345-y

5. Yuan W, Liu Z. What factors affect the quality of medical students' doctoral theses? A comparative study in affiliated hospitals of a Chinese university. Adv Med Educ Pract [Internet]. 2019 [acceso: 12/01/2021]; 10:297302. Disponible en: https://www.ncbi.nlm.nih.gov/ pmc/articles/PMC6526325/

6. Nieminen P, Sipilä K, Takkinen H-M, Renko M, Risteli L. Medical theses as part of the scientific training in basic medical and dental education: experiences from Finland. BMC Med Educ [Internet]. 2007 [acceso: 12/01/2021]; 7(1):51. Disponible en: https://pubmed.ncbi.nlm.nih. gov/18053247/

7. Soto F. Factores asociados a la elaboración de tesis en la Universidad Nacional de Asunción, Paraguay. Rev. Actual. Investig. Educ. [Internet]. 2020 [acceso: 12/01/2021]; 20(2):1-24. Disponible en: https://revistas.ucr.ac.cr/index.php/aie/article/view/41676

8. Castro-Rodríguez Y, Lara R, Pares G, Castillo S. Validez de contenido y estructura de una escala sobre limitantes para la elaboración de una tesis universitaria. Educ Med Super [Internet]. 2020 [acceso: 12/01/2021]; 34(2): e1943. Disponible en: http://scielo.sld.cu/scielo.php?script=sci_arttext $\&$ pid $=$ S0864-21412020000200009

9. Mejía CC, Cáceres OJ, Vera CA, Inga-Berrospi F, Mayta-Tristan P. Percepción y factores asociados a insatisfacción que los médicos recién graduados tienen de sus asesores de tesis, Lima-Perú. Educ Med Super [Internet]. 2016 [acceso: 14/01/2021]; 30(4):340-348. Disponible en: http://scielo.sld.cu/scielo.php?script=sci_arttext\&pi$\mathrm{d}=$ S0864-21412016000400010

10. Coruth CL, Boyd LD, August JN, Smith AN. Perceptions of Dental Hygienists About Thesis Completion in Graduate Education. J Dent Educ [Internet]. 2019 [acceso: 14/01/2021]; 83(12):1420-1426. Disponible en: https://pubmed.ncbi.nlm.nih.gov/31548304/

11. Castro-Rodríguez Y. Factores que contribuyen en la producción científica estudiantil. El caso de Odontología en la Universidad Nacional Mayor de San Marcos, Perú. Educ Med. 2019;20(s1):49-58.

12. Castro-Rodríguez Y, Sihuay-Torres K, Perez-Jimenez V. Producción científica y percepción de la investigación por estudiantes de odontología. Educ Med. 2018;19(1):1922.

13. Kim H, Sefcik JS, Bradway C. Characteristics of Qualitative Descriptive Studies: A Systematic Review. Res Nurs Health [Internet]. 2017 [acceso: 14/01/2021]; 40(1):23-42. Disponible en: https://pubmed.ncbi.nlm. nih.gov/27686751/

14. Moser A, Korstjens I. Series: Practical guidance to qualitative research. Part 3: Sampling, data collection and analysis. Eur J Gen Pract [Internet]. 2018 [acceso: 14/01/2021]; 24(1):9-18. Disponible en: https://pubmed.ncbi.nlm.nih.gov/29199486/

15. Pepin G. Reporting rigorous qualitative results: Moving beyond small sample sizes. Aust Occup Ther J [Internet]. 2018 [acceso: 14/01/2021]; 65(2):77-78. Disponible en: https://pubmed.ncbi.nlm.nih.gov/29630734/

16. Ho JCW, Wong LCJ, Wong PTP. What helps and what hinders thesis completion: a critical incident study. Int J Existential Psychol [Internet]. 2010 [acceso: 14/01/2021]; 3(2):117-31. Disponible en: http://journal.existentialpsychology.org/index.php/ExPsy/article/ view/159

17. Flynn SV, Chasek CL, Harper IF, Murphy KM, Jogensen MF. A qualitative inquiry of the counseling dissertation process. Am Counseling Assoc [Internet]. 2012 [acceso: 14/01/2021]; 51:242-55. Disponible en: https://onlinelibrary.wiley.com/doi/abs/10.1002/j.15566978.2012.00018.x

18. Nomura Y, Okada A, Miyoshi J, Mukaida M, Akasaka E, Saigo K, et al. Willingness to Work and the Working Environment of Japanese Dental Hygienists. Int J Dent [Internet]. 2018 [acceso: 14/01/2021]; 2018:2727193. Disponible en: https://pubmed.ncbi. nlm.nih.gov/30224920/

19. Kanji Z, Laronde DM. Career Outcomes of Dental Hygiene Baccalaureate Education: A Study of Graduates' Professional Opportunities, Further Education, and Job Satisfaction. J Dent Educ [Internet]. 2018 [acceso: 14/01/2021]; 82(8):809-818. Disponible en: https:// pubmed.ncbi.nlm.nih.gov/30068769/

20. Lundgren SM, Halvarsson M. Students' expectations, concerns, and comprehensions when writing theses as part of their nursing education. Nurse Educ Today [Internet]. 2009 [acceso: 14/01/2021]; 29: 527-32. Disponible en: https://pubmed.ncbi.nlm.nih.gov/19111371/

21. Barnes BJ, Williams EA, Archer SA. Characteristics that matter most: doctoral students' perceptions of positive and negative advisor attributes. Natl Acad Advising Assoc [Internet]. 2010 [acceso: 14/01/2021]; 30(1):34-46. Disponible en: https://doi.org/10.12930/0271-951730.1.34

22. Kumar S, Johnson M, Hardemon T. Dissertations at a distance: students' perceptions of online mentoring in a doctoral program. J Distanc Educ [Internet]. 2013 [acceso: 14/01/2021]; 27(1):1-15. Disponible en: http:// www.ijede.ca/index.php/jde/article/view/835 
23. Torres F. Percepción de barreras y limitantes para realizar una tesis en pregrado en estudiantes de dos facultades de medicina humana de la región de Lambayeque en el periodo agosto-noviembre 2015 [tesis de pregrado]. Lima: Universidad San Martín de Porres; 2017.
24. Nique-Carbajal C, Infante-Palacios K, Lluncor-Ushiñahua I. Limitaciones para investigar: perspectivas de los estudiantes de la Escuela de Medicina de la Universidad Católica Santo Toribio de Mogrovejo. FEM. 2020; 23(2): 95-96. 